\title{
Capital Social e Inclusión Laboral. Una aproximación a las trayectorias de ascendencia laboral de migrantes Peruanos en Chile*
}

\author{
Ignacio Madero Cabib
}

Universidad Católica, Santiago, Chile. Email: imadero@uc.cl

Claudia Mora del Valle

Universidad Alberto Hurtado, Santiago de Chile. Email: cmora@uahurtado.cl

Resumen: Este artículo analiza el fenómeno migratorio intrarregional abordando dinámicas de inclusión social informal al interior del sistema laboral. A partir del análisis de 32 relatos de vida a inmigrantes peruanos que han ascendido laboralmente en Chile, se propone que la baja ubicación de peruanos en el sistema de estratificación social chileno, condiciona su grado de dependencia a una inclusión informal al sistema laboral. Sin embargo, como efecto de la regularización de su estatus migratorio, del mayor conocimiento del campo laboral y de la formalización de su capital social, es posible el despliegue de reacciones agenciales en función de una trayectoria ascendente en el mercado del trabajo.

Palabras clave: trayectoria laboral, migrantes peruanos, capital social.

\section{Social Capital and Labour Inclusion. An approximation to the trajectories of Peruvian descent migrants working in Chile}

\begin{abstract}
In this article we analyze intraregional migratory flows focusing on dynamics of informal inclusion in the labor system. Drawing from 32 life histories of Peruvian migrants with an upward labor trajectory, we argue that a diminished place in the Chilean social stratification system conditions Peruvian migrants' dependency on informal mechanisms for inclusion in the labor system. However, as a result of the regularization of their migratory status, their increased knowledge of the labor field, and the formalization of their social capital, migrants are able to deploy agential reactions towards their upward mobility in the labor market.
\end{abstract}

Key words: employment history, Peruvian migrants, social capital.

\section{Capital Social e Inclusão do Trabalho. Uma aproximação para a trajetória de ascendência peruana, migrantes que trabalham no Chile}

Resumo: Este artigo analisa o fenômeno de migração intra-regional abordando as dinâmicas de inclusão social informal dentro do sistema de trabalho. A 
partir da análise de 32 histórias de vida de imigrantes peruanos que ascenderam profissionalmente no Chile, propõe-se que a localização baixa dos peruanos no sistema de estratificação social chilena, determina o grau de dependência de um sistema informal, incluindo o trabalho. No entanto, o efeito de regularizar sua situação migratória, um maior conhecimento do local de trabalho ea formalização de sua capital, faz possível a implantação de reações agenciais baseado em uma tendência ascendente no mercado de trabalho.

Palavras-chave: história de emprego, imigrantes peruanos, capital social.

\section{Introducción}

La migración peruana en Chile es un fenómeno social que adquirió atención sociológica hace poco tiempo atrás. La presencia de peruanos inmigrantes laborales en este país solo comienza a ser proporcionalmente considerable en la década de los 90, con la diversificación de sus países de destino a nuevas orientaciones intrarregionales, dadas las crecientes dificultades y costos de la migración al norte. Como consecuencia, las migraciones sur-sur se han establecido como una posibilidad de migración para peruanos/as de diversas condiciones económicas y sociales.

Las trayectorias laborales desarrolladas por peruanos/as en Chile, muchos con alto nivel de educación, se han caracterizado por transcurrir en un mercado laboral paralelo y secundario que concentra a estos trabajadores en un número limitado de ocupaciones. Los nichos ocupacionales de migrantes reflejan la segmentación laboral y direccionamiento que experimentan hacia actividades precarias, principalmente en el sector de servicios (Mora \& Piper, 2011; OIT, 2004; Cortés \& Groisman, 2004; KhoudourCastéras, 2007). La concentración en 'trabajos de inmigrantes’ implica generalmente un descenso laboral que acompaña la migración y que procede de las variaciones experimentadas en el tipo, estatus, y condiciones de la actividad laboral que los inmigrantes peruanos con capital cultural desempeñan en Perú y Chile.

La equivalencia existente en los sistemas laborales, por una parte, y la variación en la inclusión de los inmigrantes en las sociedades de origen y de destino, por otra, evidencian que los ordenamientos regionales y nacionales del sistema laboral varían en distintos espacios de la sociedad contemporánea. La inserción desigual en el mercado del trabajo de ambas naciones nos lleva a sostener que la equivalencia comunicativa de la sociedad mundial, es decir la existencia de un único espacio mundial con estructuras comunicativas homogéneas (Mascareño, 2010a), no es posible extrapolarla a un plano práctico u operativo, ya que las posibilidades de inclusión laboral en Chile y Perú para un peruano con alto nivel capital cultural o humano difieren drásticamente ${ }^{1}$.

La variación operativa entre sociedades, o dicho de otro modo, la existencia de una analogía comunicativa mundial que no opera de igual 
modo en la práctica, emerge vinculada al sostén normativo-social en matrices de estratificación específicas, que distribuyen jerárquicamente a los agentes en virtud de diferentes variables sociodemográficas (Grusky, 1994; Kerbo, 2005). La percepción y posesión de determinados recursos sociales afecta el modo y mecanismos de inclusión de las personas. El principio invocado es el de la plena inclusión e integración social, que puede ser entendido como la posibilidad de participación prevista para cada miembro de la sociedad (Stichweh, 2004; 2002). Desde esta perspectiva, la inclusión se refiere al modo en el que las personas son consideradas relevantes para el despliegue del funcionamiento de cada esfera social (Mascareño, 2010a, 2010b).

Este precepto de inclusión moderna no opera del mismo modo en distintas sociedades sin embargo, dado que formas de estratificación social propia de épocas anteriores no han sido eliminadas en distintas regiones del mundo, lo que constituye un obstáculo para la inclusión formal de individuos que poseen atributos socialmente desfavorables (Mascareño, 2010a). $\mathrm{Al}$ existir impedimentos para lograr una inclusión social formal y efectiva, los individuos perjudicados por el orden estratificado de la sociedad buscan integrarse a través de caminos informales o distintos a los formalmente establecidos para lograrlo (ibid). Sugerimos que esta es la vía de inclusión de los inmigrantes laborales peruanos en Chile, dado que el concurso cumulativo de jerarquías estratificadas los ubica en el camino de la inclusión informal, intensificada precisamente por la ausencia de posibilidad de inclusión formal.

Desde esta perspectiva, proponemos un análisis de los mecanismos de inclusión de inmigrantes laborales peruanos en el mercado laboral chileno, donde su posición se materializa en empleos alcanzados en virtud de criterios de selección no siempre referidos al capital cultural y humano, como la procedencia nacional por ejemplo. En primer lugar, sugerimos que la dependencia a la inclusión informal de los inmigrantes laborales peruanos en el mercado del trabajo en Chile, deviene usualmente en ocupaciones que se caracterizan por su alto grado de precariedad (Mora \& Piper, 2011). Estas condiciones de dependencia a modos informales de inclusión en la esfera laboral, implica la dependencia de los migrantes a redes sociales de reciprocidad que proporcionan información y contactos de acceso al mercado del trabajo (Mora, 2008a; Solimano 2003; Nee \& Sanders, 2001: Davila, Ghiardo \& Medrano, 2008).

En segundo lugar, exploramos en este trabajo el uso de capital social como medio de acceso y movilidad laboral entre inmigrantes peruanos que han logrado ascender ocupacionalmente en Chile. Planteamos que la dependencia a la informalidad de la integración social varía en el grado de intensidad en el caso de un inmigrante, dada la existencia de factores de estratificación que entorpecen la inclusión laboral formal (Mora, 2008b), y que por ende, la inclusión laboral es posible a través de la formalización del capital social que permite el despliegue de estrategias para lograr acceso y movilidad por vías informales. 
La conformación de nichos laborales de inmigrantes peruanos, que se ha consolidado en nuestro país, está en parte vinculada al reclutamiento laboral informal, que reproduce la segmentación en ciertos tipos de ocupaciones y actividades. Pero por otra parte, está también condicionada por la percepción de la otredad racial de los peruanos, que los direcciona a algunos empleos “de peruanos”. Esta jerarquización opera excluyendo a las personas que provienen de ese país por medio de instituciones, como el mercado laboral, que se cimientan normativamente en virtud de esta jerarquía (Mora 2008b, 2009; Mora \& Piper, 2011).

Los relatos de vida de inmigrantes peruanos en Chile que han ascendido laboralmente, evidencian que las trayectorias laborales raramente se desarrollan acorde al capital cultural que poseen. La primera inclusión laboral en Chile es generalmente informal, y en servicios caracterizados por un alto nivel de precariedad e inestabilidad. De este modo, los inmigrantes experimentan una pobreza no solamente material sino también social, es decir, ocupando una posición desfavorable e inferior en términos económicos y simbólicos (Bourdieu 1993).

\section{Migración, Diferenciación Funcional y Estratificación Social}

La región Latinoamericana evidencia interrupciones en el proceso de eliminación de la estratificación social en la modernidad (Mascareño, 2010a), dado que dentro del orden funcionalmente diferenciado permanecen redes de estratificación y reciprocidad a partir de las cuales, los individuos son evaluados según ciertas categorías sociales (clase, raza, género y etnia), que inciden directamente en las maneras en que se incluye cada uno. La migración intrarregional, específicamente el caso de los migrantes peruanos que llegan a Chile, es un ejemplo de cómo opera en la práctica la permanencia de redes de estratificación en el orden moderno.

De este modo, la mayoría de las personas de Perú que emigran a Chile se ven imposibilitadas de escalar en la estructura de oportunidades de los diferentes campos sociales dada su ubicación en distintos ejes del sistema de estratificación de este país. Los estudios que han propuesto esto (Duany, 1998, Eschbach \& Waters, 1998, De Genova, 1998, Winant, 2000, Bashi \& McDaniel, 1997, Sorenen, 2004, Held et al 2004), señalan que uno de los atributos sociales que más relevancia tiene en las trayectorias de inmigrantes corresponde a la segmentación de individuos en virtud de atributos fenotípicos y del origen nacional. Estas características son percibidas a través de un filtro social y cultural, que es utilizado tanto por las personas en la vida cotidiana como por las instituciones que poseen permanentes contactos con migrantes en Chile. El origen nacional de los migrantes, junto a procesos emergentes de racialización y la percepción que de ello se realiza en la so- 
ciedad chilena, aparecen como categorías centrales en las trayectorias migratorias de los peruanos y peruanas en nuestro país (Mora, 2009; Cordero-Guzman et al, 2001), ordenando las prácticas sociales del mercado laboral y condicionando su entrada a un estado de subordinación y marginación.

De este modo, una perspectiva de análisis que converja la diferenciación funcional y la estratificación social nos da la posibilidad de entender por una parte, la variación latinoamericana de la organización primaria de la sociedad y de modos formales e informales de inclusión (Mascareño, 2010a), específicamente en un entorno laboral. Por otra parte, nos permite observar la presencia constante de matrices de estratificación social fundadas en atributos sociales que orientan y determinan diversos procesos de la sociedad, entre los que se encuentra las dinámicas de inclusión y exclusión. De la complementación de estas aproximaciones entendemos que: a) la ubicación en el sistema de estratificación social condiciona el grado de dependencia a una inclusión informal al sistema laboral de los inmigrantes; y b) la manera en que dentro de este sistema operan actualmente criterios que son extra-laborales. Se trata entonces de estructuras de estratificación que coexisten operativamente a la diferenciación funcional de la sociedad latinoamericana, obstaculizando el pleno desarrollo del proceso de inclusión social, lo que conlleva reacciones agenciales de los inmigrantes, condicionadas por su acceso y manejo de conocimiento del campo laboral chileno y el uso de sus capitales.

\section{Reacciones Agenciales a los procesos de Estratificación}

Del análisis de la forma de inclusión y exclusión existente en el mercado laboral chileno, emerge la pregunta sobre el despliegue de mecanismos, procesos y condiciones detrás de trayectorias laborales ascendentes de inmigrantes peruanos, en un contexto en el cual gran parte de sus connacionales no las exhiben. Dicho en los términos discutidos anteriormente, la interrogante se dirige a la capacidad de evaluación de modos informales de integración laboral de migrantes peruanos en nuestro país y a las capacidades agenciales de los migrantes para soslayar en sus trayectorias de vida el efecto pernicioso del contexto laboral estratificado y racializante.

Esta influencia condicional del mercado laboral está sujeta, sin embargo, a la evaluación reflexiva de los inmigrantes, que la ponderan en relación a sus capacidades, en relación a sus otras preocupaciones, y en razón de sus propias propiedades emergentes de autoconciencia y automonitoreo (Archer, 2009). En este sentido, existe la opción de que los mismos migrantes eludan la constricción del entorno institucional y a la vez habiliten nuevas posibilidades de ascendencia laboral en virtud de las tácticas o estrategias que cada uno de ellos emplee, como una forma efectiva de paulatinamente sobrepasar los obstáculos que se es- 
grimen desde la estructura del campo laboral. El uso de una estrategia o táctica varía en función de la posesión de poder sobre un espacio social determinado. De tal manera, mientras la táctica se encuentra determinada por la ausencia de poder del individuo dado un contexto perjudicial que le impide actuar en función de sus propias deliberaciones, la estrategia se encuentra organizada por el principio del poder efectivo de cada sujeto (De Certeau, 2000).

Para el caso de los inmigrantes, este principio de poder para desplegar estrategias se traduce en primer lugar, en la posibilidad de regularización de su estatus migratorio para acceder a los beneficios de ciudadanía. En segundo lugar, en el conocimiento y manejo de mecanismos de acceso al campo laboral chileno. Y, en tercer lugar, en la formalización de su capital social, vale decir, el contacto y la asociación con personas y asociaciones con recursos potencialmente beneficiosos para su trayectoria (Bourdieu, 2000). De este modo, la formalidad y la informalidad están condicionadas no solamente por el mecanismo de inclusión al mercado laboral sino que además, por el tipo de red social de cada inmigrante, todo lo cual incide en la selección de estrategias o tácticas para acceder al campo laboral chileno.

En los párrafos siguientes nos enfocaremos en los procesos que posibilitan trayectorias laborales ascendentes de peruanos en Chile, en particular la informalidad del acceso al mercado del trabajo y su condicionamiento por jerarquías sociales que impiden su inclusión formal. En segundo lugar, exploraremos las reacciones agenciales, es decir estrategias y tácticas (De Certeau, 2000) que los inmigrantes laborales peruanos generan para lograr su integración en el mercado laboral chileno.

La metodología utilizada en este trabajo corresponde a la emergente "Metodología de sistemas fundamentada" (Flores, 2009), dirigida a generar conocimiento empírico sobre mecanismos operativos de sistemas de la sociedad a partir de las percepciones de individuos que realizan observaciones parciales. La técnica de levantamiento de datos seleccionada es el relato de vida, pues contribuye a capturar el sentido de los recorridos laborales de los inmigrantes en su contexto cultural e histórico especifico. La muestra para esta investigación (reportada en Tabla 1) consiste en 32 relatos de vida de inmigrantes peruanos con una trayectoria laboral en Chile que haya incluido al menos dos trabajos, el último de los cuales debió implicar un ascenso en ingresos o estatus. Los relatos de vida se realizaron entre el mes de septiembre y noviembre de 2010 y fueron analizados narrativamente. 
Tabla 1: Categorización de participantes en relatos de vida

\begin{tabular}{|c|c|c|c|}
\hline Categoría & Género & $\begin{array}{l}\text { Entrevistados/as } \\
\text { con Visa Definitiva }\end{array}$ & $\begin{array}{c}\text { Entrevistados/as } \\
\text { con nivel } \\
\text { educacional } \\
\text { superior completo o } \\
\text { incompleto }\end{array}$ \\
\hline & 17 Hombres & 16 Hombres & 13 Hombres \\
\hline & 15 Mujeres & 15 Mujeres & 11 Mujeres \\
\hline Total & 32 & 31 & 24 \\
\hline
\end{tabular}

Fuente: Elaboración propia.

\section{Dependencia a formas de integración informales en el mercado laboral chileno}

El acercamiento al mercado laboral nacional de los inmigrantes peruanos está caracterizado por las escasas oportunidades ofrecidas y por condiciones de precariedad laboral. La percepción sobre el direccionamiento hacia 'labores de inmigrantes' o nichos ocupacionales, ha sido recogida por distintos estudios respecto de los empleadores de migrantes peruanos y evidenciada por los inmigrantes peruanos entrevistados, quienes afirman que en Chile la mayoría de los peruanos están destinados a ocupar plazas de trabajo que se caracterizan por ser labores inestables y de bajo estatus (Hill Maher \& Staab, 2005; Mora, 2011).

La primera inserción al mercado del trabajo chileno constituye para la gran mayoría de los inmigrantes, una inclusión informal en labores que encuentran con la colaboración de personas u organizaciones que trabajan con inmigrantes y que poseen recursos y contactos beneficiosos para asegurar un empleo, pero no para trabajos acordes a su capital cultural. En efecto, el acceso segmentado al mercado laboral de los inmigrantes está determinado en parte por la estructura informal de reclutamiento de trabajadores y el rol de las redes establecidas, que canalizan la oferta de empleo hacia nichos ocupacionales específicos (Pessar y Mahler, 2006; Pedraza, 1991). Es a través de redes de parientes, amigos y connacionales que proporcionan contactos y recomendaciones que los inmigrantes encuentran oportunidades laborales, lo que reproduce su segregación en ciertos tipos de empleo. Las redes migratorias contribuyen a consolidar la segmentación ocupacional en la medida que actúan como agentes informales de colocación de empleo en trabajos con bajas pretensiones salariales y en los cuales no son integrados en función de sus habilidades. 
La dificultad para convalidar títulos técnicos y profesionales y la lentitud de la gestiones para adquirir la ciudadanía definitiva, inciden también en que los migrantes accedan en primera instancia a un trabajo no vinculado a su capital cultural. Aún aquellos que han tenido vasta experiencia laboral en Perú, declaran que al momento de la entrada al mercado laboral chileno es impensable exigir mejores trabajos o condiciones laborales. El estar incluidos en el sistema laboral en condiciones inestables es significado por algunos migrantes como algo usual (pero injusto), dada la vulnerabilidad social del primer período migratorio. Así, en el primer contacto con la institucionalidad en Chile, se genera una disgregación entre las expectativas laborales que poseían al momento de llegar al país y la experiencia de ingresar a trabajos caracterizados por un alto grado de vulnerabilidad social. Al respecto Pedro (nombre ficticio), profesor de música en colegios privados en Lima, residente hace 7 años en Chile sostiene:

“Toda mi vida he venido de vacaciones acá a Chile. Pero nunca pensé venirme a vivir y menos casarme. Y entonces me gustaba Chile desde antes. Quería trabajar al principio en lo mismo, como profe de música, pero es un poco difícil porque tengo que reconvalidar, y existe esa posibilidad pero es caro, pero si se puede. Me dijeron que cuesta más de un millón. Y aparte hay que tener el tiempo y la plata de la familia, entonces comencé a trabajar en este rubro ¿̇no?, en servicios [conserjería]”.

Pedro, tal como otros inmigrantes, tras muchos meses de búsqueda de empleo como profesor de música en diversos colegios de Santiago, solo logró integrarse como jefe de conserjes en un edificio de la comuna de Providencia. De este modo, las expectativas de desarrollar carreras académicas o de generar negocios en Chile son desmoronadas al ver que los mecanismos de inclusión en el mercado laboral promueven oportunidades limitadas para acceder a ocupaciones en las que puedan llevar a cabo sus proyectos profesionales o comerciales.

Sin embargo, luego de alcanzar una condición regular o documentada en Chile, de obtener conocimiento del campo laboral en nuestro país, y de generar redes profesionales o comerciales relevantes al campo, los migrantes generalmente son capaces de demandar derechos migratorios, principalmente en términos laborales, y de diversificar las fuentes ocupacionales (Mora \& Piper, 2011). Resulta frecuente observar que una vez adquirida estas condiciones, existan proyectos de estudio, de profesionalización o de expansión comercial. Es el caso de Ana (nombre ficticio), quien al llegar a Chile hace 12 años atrás, comenzó desempeñándose como temporera durante 3 años y como asesora del hogar otros 6 años. En el desarrollo de su trayectoria, el contacto con diferentes actores sociales que se encontraban en una posición favorable en diversos contextos laborales, la llevó a concentrar sus expectativas en el acceso a una posición laboral acorde a sus intereses iniciales. Para ello, además de regularizar su situación legal en Chile, Ana a través del préstamo de conocidos peruanos y del ahorro sistemático de una proporción de su sueldo, logró 
montar un cibercafé en el centro de Santiago. Respecto a sus expectativas de movilidad laboral, actualmente señala:

"Quisiera explorar otros rubros, o sea mi sueño es tener una pollería aquí, vamos a ver si lo puedo lograr. Una pollería como un restaurante de pollo peruano. Eso es lo que no se, lo quiero hacer. Y dentro de ello ya viene la gastronomía peruana, pero por lo menos que no sean de clase alta, sino de clase media para que se pueda consumir mas que todo, precios accesibles ¿̇me entiendes?”

Es a través de la formalización del capital social que los inmigrantes como Ana derivan generalmente su integración al mundo del trabajo. Las dinámicas de inclusión informal al sistema laboral chileno vivenciada por migrantes peruanos advierten que este sistema no posee suficientes mecanismos para proveer una oportunidad de integración a todos los individuos de un modo regular o institucional. La carencia de mecanismos de inclusión formal al espacio laboral chileno de los inmigrantes, muestra las limitaciones de este campo para generar igualdad funcional a todos los individuos que participan en él y generan mecanismos informales donde el capital social es fundamental en el logro de la inclusión. Ello sólo es posible a través de la acumulación de experiencia migratoria, que conlleva además el reconocimiento de la necesidad de regularización de estatus y un manejo del campo laboral.

La institución del mercado laboral no posee el incentivo para generar universalidad de inclusión, lo que produce que el grado de dependencia a la inclusión informal al trabajo sea mayor para peruanos dado que enfrentan mayores barreras de integración por el efecto de su ubicación en jerarquías sociales. Esto evidencia una incapacidad del sistema laboral-económico de este país para generar la inclusión suficiente y necesaria para los y las migrantes peruanos/as. Planteamos esto no sólo como una crítica normativa a la informalidad de la inclusión laboral de los inmigrantes, sino como una advertencia general sobre el sistema laboral chileno como entidad que no ha logrado desarrollar mecanismos abiertos, universalistas o cosmopolitas de inclusión social. La crítica no es simplemente hacia la manera en que se articulan modos informales de inclusión laboral, sino al modo en que no se integran operativa y comunicacionalmente a la sociedad las demandas de mayor integración formal por parte de inmigrantes peruanos, que normalmente vivencian situaciones caracterizadas por la vulnerabilidad y la exclusión social.

\section{Formalización del capital social en la generación de tácticas y estrategias agenciales para una trayectoria laboral ascendente}

La capacidad de reacción para eludir los obstáculos del mercado laboral, se vinculan con el grado de formalización del capital social de los inmigrantes. Así, en los relatos de los entrevistados para este estudio, emerge 
un vínculo entre la formalidad de la red social en un campo laboral determinado y la posibilidad de cada migrante para acceder a trabajos en función de su capital cultural y humano, y no de criterios diferentes. De este modo, las formas (tácticas y estrategias) con que los inmigrantes logran evadir las trabas institucionales que encuentran en Chile, varían en similar orientación que el aumento de conocimiento del contexto laboral, la regularización de su estancia en Chile y la formalización de su capital social en sus respectivos campos laborales.

A partir de la muestra investigada en este estudio, sugerimos que el uso de tácticas -o reacciones desde la falta de un lugar propio (De Certeau, 2000)- se vincula con un estatus migratorio inconcluso o no definitivo, con una baja información sobre derechos y conocimiento del campo laboral, y con la posesión de redes de instituciones y personas con niveles precarios de capital cultural y material. Dichas condiciones constriñen la capacidad agencial a acciones de mediano alcance y de menor impacto en las posibilidades de una movilidad laboral ascendente de los inmigrantes laborales peruanos en Chile.

Entre los ejemplos de tácticas más recurrentes se encuentran: a) Estimar y aprovechar instrumentalmente la amabilidad de personas de nacionalidad chilena para efectos laborales. b) Buscar incluirse en primera instancia en cualquier trabajo, pero sentir siempre que el trabajo es digno, pues la seriedad de una labor depende del valor que cada migrante le dé. c) Querer distinguirse cotidianamente de los y las migrantes irresponsables, 'corto-placistas' en sus objetivos, involucrados en vicios, para establecer una distinción simbólica con ellos y demostrar la seriedad de sus proyectos personales. d) Tener la disposición a trabajar por sueldos bajos, permanecer horas extras y poseer la capacidad de tolerancia, pasividad y resiliencia frente a conflictos laborales. e) Poseer todos los documentos de extranjería actualizados. f) Crear confianza en los empleadores y clientes y establecer una buena reputación. g) Incorporar los códigos lingüísticos (Berstein, 1961), es decir, los modos de expresarse del campo laboral en que cada uno se encuentre. h) Y finalmente, aferrarse a una noción de religiosidad (Weber, 2006) que otorgue la confianza individual para mantener la esperanza de que su situación material en Chile puede mejorar.

Este tipo de tácticas se despliegan usualmente en contextos en los cuales la presencia de redes sociales informales, entendidas como la asociación con personas con bajo capital cultural y baja influencia en su entorno laboral, impide el logro de expectativas laborales iniciales de los inmigrantes y obliga a desempeñarse en labores inconsistentes a su capital humano y cultural. Es la formalización del capital social, definido como la obtención de un conjunto de recursos simbólicos (actuales o potenciales) (Bourdieu, 1980), que reportan a un individuo un beneficio económico y cultural, lo que permite la anulación de los efectos de la estratificación de la sociedad en su inserción y trayectoria laboral. 
En otras palabras, la formalización del capital social posibilita que un sujeto sea capaz de promover acciones y movilizarse para alcanzar un objetivo que disminuya los obstáculos que emergen del orden estratificado de la sociedad. Los inmigrantes que logran formalizar su capital social y beneficiarse de los recursos materiales y simbólicos que le proporcionan sus redes compuesta de personas y organizaciones con incidencia en sus entornos laborales y comerciales, habilitan sus posibilidades de acción hacia estrategias para lograr una movilidad laboral.

Las estrategias que emergen de los relatos de vida de inmigrantes laborales peruanos comprenden: a) Interés por estudiar una nueva carrera universitaria en Chile. b) Deseo de profesionalizar los servicios entregados. c) Proyecciones de ampliación del negocio en el rubro en que se desenvuelven, e incursionar en nuevos proyectos. d) Tener la capacidad para proyectarse y, en consecuencia generar objetivos a largo plazo manteniendo prácticas cotidianas coherentes a ellos como por ejemplo, gastar el mínimo en comodidades, o evitar tener hijos. e) Endeudarse con créditos bancarios para poder llevar a cabo proyectos comerciales o carreras universitarias.

La distinción que realizamos entre tácticas y estrategias no implica sin embargo, que su uso sea excluyente. Frecuentemente un inmigrante pasa de la utilización de unas a otras. No obstante, en gran parte de los casos se observa que el manejo de tácticas siempre es previo a las estrategias. Esto, por efecto del conocimiento adquirido del campo laboral, la paulatina incorporación de códigos culturales, la regularización de su condición migratoria y la formalización de su capital social. Además, tanto los inmigrantes que solo pueden llevar cabo tácticas como los que regularmente esgrimen estrategias sostenibles, intentan mantener, formalizar e institucionalizar cada vez más sus redes sociales, es decir, velan constantemente por confeccionar una red de organizaciones y personas que les permita asegurar la integración al mercado laboral en ocupaciones acordes a su capital cultural. De esta manera, los inmigrantes centralizan sus acciones individuales en la disipación de las barreras de inclusión al sistema laboral generadas por la estratificación de la sociedad.

Con todo, los efectos de las matrices de estratificación social en el acceso al sistema del trabajo no sólo afecta los inmigrantes peruanos ni es algo exclusivo de Chile. Lo que sugerimos en este trabajo es que la jerarquización de los inmigrantes en virtud de su origen nacional, clase y género, inciden en el grado o intensidad en que un individuo es incluido en un contexto social de un modo informal. La experiencia de los inmigrantes peruanos en el sistema laboral chileno evidencia que el mercado del trabajo presenta obstáculos derivados de la matriz de estratificación, incluyendo los que han experimentado una ascendencia laboral. Para todos ellos, la inclusión laboral se caracteriza por su informalidad y su trayectoria ascendente, por la formalización de su capital social. 


\section{Conclusión}

El fenómeno migratorio intrarregional y específicamente el caso de los inmigrantes peruanos en Chile, es un caso de estudio privilegiado para evidenciar el ordenamiento descontinuado de la diferenciación funcional en América Latina, a propósito de la creación de jerarquías sociales que coexisten al proceso de organización primaria de la sociedad moderna (Mascareño, 2010a). Hemos argumentado que la permanencia de matrices de estratificación social afectan los procesos de inclusión de migrantes peruanos en Chile, pues contribuyen a la generación de maneras de integración a la sociedad distintas a las formalmente establecidas. Ello porque el posicionamiento de los inmigrantes en lo más bajo de la estructura social, condiciona sus oportunidades de vida y sus trayectorias laborales, orientándolos a utilizar vías informales de inclusión al mercado del trabajo (Mora, 2009).

De este modo, en el presente artículo hemos enfatizado la existencia de dinámicas de inclusión social que en ciertos contextos sociales (como la esfera laboral) de Latinoamérica están determinadas por efectos del sistema de estratificación. Este acercamiento nos permite observar manifestaciones de estratificación social al interior del mercado del trabajo y establecer que las posibilidades de inclusión laboral, formal e informal, actualmente se encuentran permeadas por dichas jerarquías. En este sentido, se ha sugerido que incluso los inmigrantes peruanos que ascienden laboralmente en nuestro país están subordinados a criterios extra-laborales, lo que deriva en su dependencia de la formalización de capital social como mecanismo de inclusión.

La segmentación laboral de los inmigrantes peruanos en nichos precarios independiente de su capital cultural, da cuenta de la incapacidad del sistema laboral de generar mecanismos abiertos de inclusión y aún más, la inclusión informal de migrantes que ingresan por primera vez al mercado laboral chileno se convierte en un pilar de legitimación de la acción del sistema laboral (Mascareño, 2007; Ottone et al, 2007). Existe una cierta estabilización en la direccionalidad que asume la forma de operación del sistema laboral chileno, caracterizada por diferenciar el origen nacional y percepción racial de las personas, a tal punto que hasta los migrantes peruanos que ascienden laboralmente en nuestro país deben vivenciarla.

En Chile se ha edificado una forma particular de estratificación que afecta generalmente a los inmigrantes peruanos (Mora 2008b, 2009; Mora y Piper, 2011). Aquí, el origen nacional, la clase, la etnia, el género o la percepción racial operan como obstaculizadores de la inclusión laboral, dificultando el acceso a ocupaciones coherentes con el capital cultural de cada persona (Mora, 2009; Cordero-Guzmán et al, 2001). Sin embargo, como plantea Archer (2009), los inmigrantes son portadores de una capacidad agencial que logra traspasar las constricciones institucionales, generando posibilidades de movilidad ocupacional a través del despliegue de tácticas y estrategias para eludir las barreras presentadas a los mecanismos 
de inclusión. La evaluación reflexiva de los inmigrantes es el antecedente necesario. La ascendencia laboral de los inmigrantes puede tener lugar cuando acciones y reacciones se despliegan ante el reconocimiento de oportunidad de inclusión al mercado del trabajo. El siguiente paso, es la exploración de las circunstancias objetivas y subjetivas que facilitan la apertura de esta condición de posibilidad. 
Polis, Revista de la Universidad Bolivariana, Volumen 10, $N^{\circ} 29,2011$

\section{Notas}

* Investigación financiada por FONDECYT N¹100793.

${ }^{1}$ El capital cultural y humano da cuenta del nivel académico adquirido por los migrantes en universidades o centros técnicos y además, de las disposiciones e inclinaciones adquiridas a propósito de sus contextos familiares o laborales anteriores (Bourdieu, 2000). 


\section{Bibliografía}

Archer, Margaret (2009), Teoría Social Realista: El Enfoque Morfogenético, Traducción Daniel Chernillo, Universidad Alberto Hurtado, Santiago de Chile.

Bashi, Vilna \& McDaniel Antonio (1997), “A theory of immigration and racial stratification”, en Journal of Black Studies N5(27).

Berstein, Basil (1961), "Social class and linguistic development: a theory of social learning”. En: Halsey, A. H. Floud, J. Anderson, C. A. (comps), Education, economy and society, Free Press, Nueva York.

Bourdieu, Pierre (1980), "Le Capital Social”, en Actes de la recherche en Science Sociales №31.

Ídem (1993), La miseria del mundo, Akal, Barcelona.

Ídem (2000), Poder, derecho y clases sociales, Desclée de Brouwer, Bilbao.

Cordero-Guzmán, Héctor; Smith, Robert \& Grosfoguel, Ramón (2001), Migration, Transnationalization, and Race in a Changing New York, Temple University Press, Philadelphia.

Cortés, Rosalía \& Groisman, Fernando (2004), “Migraciones, mercado de trabajo y pobreza en el Gran Buenos Aires”, en Revista CEPALN82.

Dávila, Óscar; Ghiardo, Felipe \& Medrano, Carlos (2008), Los desheredados. Trayectorias de vida y nuevas condiciones juveniles, CIDPA, Valparaíso.

De Certau, Michel (2000), La invención de lo cotidiano: Artes de Hacer, Universidad Iberoamericana, México D.F.

De Genova, Nicholas (1998), "Race, Space, and the Reinvention of Latin America in Mexican Chicago”, en Latin American Perspectives N5 (25).

Duany, Jorge (1998), "Reconstructing Racial Identity. Ethnicity, Color, and Class among Dominicans in the United States and Puerto Rico”, en Latin American Perspectives $\mathrm{N}^{\circ} 3$ (25).

Eschbach, K \& Waters, Mary (1995), “Immigration and Ethnic and Racial Inequality in the United States”, en Annual Review of Sociology 21.

Flores, Rodrigo (2009), Observando Observadores, Universitaria, Santiago de Chile.

Grusky, David (1994), Social Stratification in Sociological Perspective: 
Class, Race, and Gender, Westview, Boulder.

Held, David; McGrew, Anthony; Goldblatt, David \& Perraton, Jonathan (2001), Transformaciones Globales: Política, Economía y Cultura, Oxford University Press, México D.F.

Hill- Maher, Kristen, Staab, Silke (2005), “Nanny Politics. The Dilemmas of Working Women's Empowerment in Santiago”, en International Feminist Journal of Politics $\mathrm{N}^{\circ} 7$.

Kerbo, Harold (2005), Social Stratification and Inequality, class conflict in historical, comparative, and global perspective, McGraw Hill, New York.

Khoudour-Castéras, David (2007), “Migraciones internacionales y desarrollo: el impacto socioeconómico de las remesasen Colombia”, en Revista CEPAL, $\mathrm{N}^{\circ} 92$.

Mascareño, Aldo (2007), “Cultura como ficción real”, en Vicuña, Manuel (ed.): El chile del bicentenario: Aportes para el debate, Universidad Diego Portales, Santiago de Chile.

Ídem (2010a), Diferenciación y Contingencia en América Latina, Universidad Alberto Hurtado, Santiago de Chile.

Ídem (2010b), “Coordinación social mediante políticas públicas: el caso chileno”, Revista CEPAL N¹01.

Mora, Claudia (2008a), “Globalización Género y Migraciones”, en Revista Polis $\mathrm{N}^{\circ} 20$, Universidad Bolivariana.

Ídem (2008b), "The Peruvian comunity in Chile as a response to discrimination and exclusion”. En Peace $\mathrm{N}^{\circ} 3(20)$ Special Issue on Citizenship and Social Justice.

Ídem (2009), “Estratificación Social y Migración Intrarregional: Algunas caracterizaciones de la experiencia migratoria en Latinoamérica”, Revista Universum, Enero.

Ídem (2011), “Global Inequalities - Local Hierarchies.Peruvian Migrants’ Labor Niches and Occupational Mobility in Chile”, en Globalization and Inequality in Emerging Societies, Ed. BoikeRehbein, Palgrave Mcmillan, Hampshire.

Mora, Claudia \& Piper, Nicola (2011), "Peruvian Female Workers in Chile: the Migrant Experience and Migrant Rights”, en Diversities N¹(13).

Nee, Victor \& Sanders, Jimy (2001), “Understanding the diversity of immigrant incorporation: a forms-of-capital model”, en Ethnic and racial studies $\mathrm{N}^{\circ} 3(24)$. 
OIT (2004), En busca de un compromiso equitativo para los trabajadores migrantes en la economía globalizada, Conferencia Internacional del Trabajo $92^{\mathrm{a}}$ reunión, Ginebra.

Ottone, Ernesto; Sojo, Ana, Espíndola, Ernesto, Feres, Juan, Hopenhayn, Martín, León, Arturo, Uthoff, Andras \& Vergara, Carlos (2007), Cohesión Social: Inclusión y sentido de pertenencia en América Latina y el Caribe, CEPAL División de Desarrollo Social, Santiago de Chile.

Pedraza, Silvia (1991), "Women and Migration: The social consequences of Gender”, en Annual Review of Sociology 17.

Pessar, Patricia \& Mahler, Sarah (2006), “Transnational Migration: Bringing Gender In”, en International Migration Review N³(37).

Solimano, Andrés (2003), “Globalización y migración internacional: la experiencia latinoamericana”, en Revista de la CEPAL N80.

Sorensen, Ninna (2004), “Globalización, Género y Migración Transnacional. El Caso de la Diáspora Dominicana”, en: Migración y desarrollo, CSIC, Buenos Aires.

Stichweh, Rudolf (2004), On the Genesis of World Society: Innovations and Mechanisms, Luzern, Universität Luzern, Luzern.

Ídem (2002), “Strangers, Inclusions and Identities”, en Soziale Systeme N॰8 (1).

Weber, Max (2006), La ética protestante y el espíritu del capitalismo, Terramar, Buenos Aires.

Winant, Howard (2000), "Race and Race Theory", en Annual Review of Sociology 26.

Recibido: 25.05.2011

Aceptado: 06.07.2011 